\title{
Machine Learning for Additive Manufacturing of Electronics
}

\author{
Stoyan Stoyanov and Chris Bailey \\ Computational Mechanics and Reliability Group (CMRG) \\ University of Greenwich, London SE10 9LS, United Kingdom \\ Email: s.stoyanov@gre.ac.uk
}

\begin{abstract}
Quality of electronic products fabricated with additive manufacturing (AM) techniques such as $3 D$ inkjet printing can be assured by adopting pro-active predictive models for process condition monitoring instead of using conventional post-manufacture assessment techniques. This paper details a model-based approach, and associated machine learning algorithms, which can be used to achieve and maintain optimal product quality during production runs and to realise model predictive process control $(M P C)$. The investigated data-driven prognostics based on state-space modelling of the dynamic behaviour of $3 D$ inkjet printing for electronics manufacturing is new and makes it an original contribution. $3 D$ printing of conductive lines for electronic circuits is a main targeted application, and is used to demonstrate and validate the prognostics capability of machine learning models developed from measured process data. The results show that, for moderately non-linear dynamics of the $3 D$ Printing process, state-space models can inform on the expected process trends (states) and related product quality characteristics even over large prediction horizons. The models can also support the realisation of model predictive process control for optimal target performance.
\end{abstract}

\section{INTRODUCTION}

The past few years have marked a significant growth and developments of 3D-Printing (more broadly Additive Manufacturing, AM) for digital manufacture of electronics products driven by the benefits and advantages which these types of technologies can offer. While current use can be found predominantly in the area of physical prototyping, there is an increasing interest and shift towards adoption of additive manufacturing techniques within production lines. The main drivers for the industry to consider and adopt additive manufacturing for their products are the design flexibility, the high levels of product customisation at relatively low cost and the shorter lead times.

There are 7 main types of additive manufacturing technologies defined in the Standard F2792 produced by the American Society for Testing and Materials (ASTM) [1]. Within these process types of additive manufacturing, examples of technologies that have gain popularity and are now used in different applications include fused deposition modelling (FDM), selective laser sintering, inkjet printing and stereolithography. Figure 1 details the main process types in additive manufacturing according to F2792 standard.

\begin{tabular}{|c|c|c|c|}
\hline \multicolumn{2}{|r|}{ Process Type } & Description & Related Technologies \\
\hline$\# 1$ & Material Extrusion & $\begin{array}{l}\text { Material is selectively dispensed through a } \\
\text { nozzle or ban orifice }\end{array}$ & Fused deposition modelling \\
\hline$\# 2$ & Material Jetting & $\begin{array}{l}\text { Droplets of build material are selectively } \\
\text { deposited }\end{array}$ & $\begin{array}{l}\text { Ink-jet printing } \\
\text { Multi-jet-modelling }\end{array}$ \\
\hline$\# 3$ & Powder bed fusion & $\begin{array}{l}\text { Thermal energy selectively fuses regions of } \\
\text { powder bed }\end{array}$ & $\begin{array}{l}\text { Selective laser sintering (SLS) } \\
\text { Electron beam melting (EBM) }\end{array}$ \\
\hline$\# 4$ & $\begin{array}{l}\text { Directed energy } \\
\text { deposition }\end{array}$ & $\begin{array}{l}\text { Focused thermal energy used to fuse materials } \\
\text { by melting as material is deposited }\end{array}$ & Laser metal deposition (LMD) \\
\hline$\# 5$ & Vat Photopolymerisation & $\begin{array}{l}\text { Liquid photopolymer in a vat is selectively cured } \\
\text { by light-activated polymerisation }\end{array}$ & $\begin{array}{l}\text { Stereolithography (SLA) } \\
\text { Digital Light Processing (DLP) }\end{array}$ \\
\hline$\# 6$ & Binder jetting & $\begin{array}{l}\text { Focused thermal energy used to fuse materials } \\
\text { by melting as material is deposited }\end{array}$ & $\begin{array}{l}\text { Powder bed and Ink-jet head (PHID) } \\
\text { Plaster-based 3D printing (PP) }\end{array}$ \\
\hline$\# 7$ & Sheet Lamination & Sheets of a material are bonded to form 3D-part & $\begin{array}{l}\text { Laminated object Modelling (LOM) } \\
\text { Ultrasonic consolidation (UC) }\end{array}$ \\
\hline
\end{tabular}

Fig. 1. Additive manufacturing process types.

Different additive manufacturing technologies have different advantages/disadvantages in terms of materials that can be processed, speed of fabrication, dimensions and tolerances that can be achieved, etc. Although these attributes change and mature continually, the wider adoption of 3D-Printing as a viable technology in electronic manufacture requires that present challenges related to performance, quality and reliability of printed electronics are successfully addressed. Given the layer-by-layer principle of building the physical domain of the product, the most critical quality issue with 3D-Printing is the dimensional/shape accuracy of the printed structures and conforming to required specifications (e.g. resistivity requirements for conductive lines fabricated 
with 3D inkjet printing). Residual stress build-up in the layered structure that can compromise the structural stability and mechanical performance (e.g. warpage, residual deformation, etc.) are important reliability issues of concern.

Computational intelligence techniques such as artificial neural networks, fuzzy systems and genetic algorithms have been used for quality prediction in a variety of applications but so far have found limited use in relation to $3 \mathrm{D}$ printing processes for digital electronics manufacturing. The advantages of forecasting the printing process dynamics using suitable machine learning process models are discussed in this paper. Such approach is shown to be highly beneficial as it can improve the quality of 3D fabricated electronics parts and packages, for example in the context of dimensional accuracy. It can also support the realisation of in-line process control capability by means of model predictive control.

\section{Status of Printing for EleCtronics}

Printing for electronics in general and for electronics packaging in particular is not new but has seen growth in the past years. Use of printing processes can be found in a range of applications including smart packaging, medical devices, active clothing and sensors. Examples of manufacture using printing processes are reported for thin-film transistors, conductive and photovoltaic elements of electronics packages, interconnects, MEMS and sensors [2-5]. Selected examples of printed products are illustrated in Figure 2.

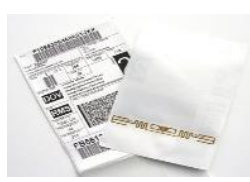

RFID labels

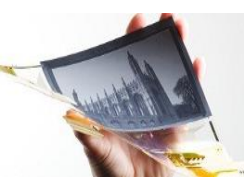

Flexible Displays

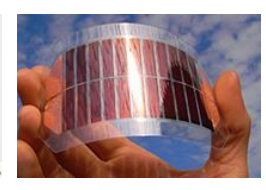

Photovoltaics
Fig. 2. Electronics products manufactured using printing processes.

3D-Printing aims to deliver opportunities for electronics manufacturing in a truly $3 \mathrm{D}$ manner. For example, in packaging this includes the circuitry and the package structure within which electrical components are embedded as the package is built. There is also stronger focus on the capability of using multiple materials (dielectric, conductive and functional). For example, Voxel8 in USA are marketing their 3D printer for electronics with capability to build 3D electronics products through FDM, component placement and printing conductive inks [6]. The European consortium behind the NextFactory project is developing an all-in-one printing platform for electronics manufacture and packaging that combines modules for multi-material 3D inkjet printing, material cure/sintering, component assembly and in-line inspection [7].

\section{State-Space Modelling ANd Model Predictive Control (MPC)}

\subsection{State-Space Model Identification}

System model identification using measured data is an experimental approach to determine a parameterised model for the dynamical behaviour of an industrial process by using respective input signals [8-10]. The parameterised model structure that needs to be identified, $M(\theta)$, has a predefined form where $\theta=$ $\left(\theta_{1}, \theta_{2}, \ldots, \theta_{d}\right) \in R^{d}$ is the parameter vector of the unknown model parameters that require estimation. The development of a discrete linear state-space model, defined in the so called innovations form, requires identification of the system when the model structure has the following form:

$$
\begin{gathered}
x(t+1)=A(\theta) x(t)+B(\theta) u(t)+K(\theta) e(t) \\
y(t)=C(\theta) x(t)+e(t)
\end{gathered}
$$

where $u(t) \in R^{m}, y(t) \in R^{p}$ and $e(t) \in R^{p}$ are the system input, output, and disturbance (measurement noise) vectors at time $t$, respectively. The state of the system is represented by the column vector $x(t) \in R^{n}$ which has the dimension of the model order (i.e. $n)$. The matrices $A(\theta) \in R^{n \times n}, B(\theta) \in R^{n \times m}, C(\theta) \in R^{p \times n}$ and $K(\theta) \in R^{n \times p}$ are the state-space linear model matrices. Their unknown elements, for a particular model structure (e.g. canonical form where $A, B$ and $C$ are parameterised in a canonical form, as described in [8]), constitute the vector $\theta \in R^{d}$. A diagram of the state-space model structure is illustrated in Figure 3.

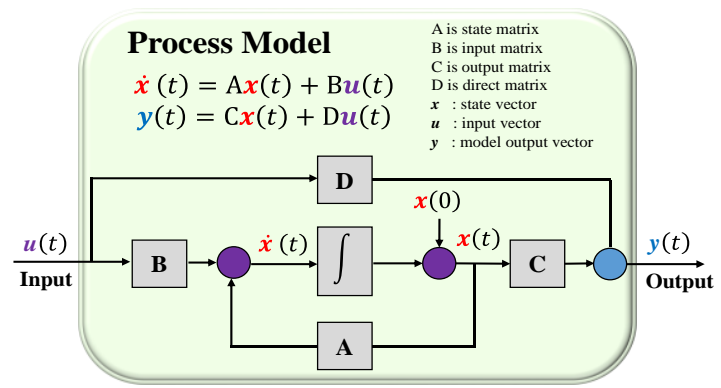

Fig. 3. State-space model structure. 
Most system identification techniques, including in the case of state-space model identification, are based on minimization of the prediction error (PEM) in the space of the model structure defined with the model parameter vector $\theta$ [8]. Given the availability of estimation data that consist of an input sequence

$$
\{u(t) \mid t=1,2, \ldots, N\}
$$

and an output sequence

$$
\{y(t) \mid t=1,2, \ldots, N\},
$$

the vector of the prediction error can be defined as

$$
e(t, \theta)=y(t)-\hat{y}(t \mid \theta)
$$

where $\hat{y}(t \mid \theta)$ is the predictor given by

$$
\hat{y}(t \mid \theta)=C(\theta) x(t)
$$

This is computed using known signals $u$ and $y$ up to discrete time $t=N$. The prediction error $e(t, \theta), t=1,2, \ldots, N$ is the difference between the measured output and the predicted output of the model at discrete time $t$. Therefore, the system identification of the state space model requires to find out a model parameters vector $\hat{\theta}_{N}$ that minimises the prediction error, i.e.

$$
\hat{\theta}_{N}=\min _{\theta} V_{N}(\theta)
$$

where

$$
V_{N}(\theta)=\frac{1}{N} \sum_{t=1}^{N}|e(t, \theta)|^{2}
$$

and $|\cdot|$ is the Euclidian $\mathrm{L}_{2}$-norm.

It should be noted that the identification of the model parameters uses an initial estimate for the state of the system. Algorithm realisations typically assume an initial state vector $x(0)$ with zero elements or estimate the initial state by treating the values of $x(0)$ as independent parameters along with the model parameters during the PEM procedure.

There are also subspace state-space system identification methods such as the popular 4SID which does not require priori model parameterisation and subsequent non-linear optimisation. These methods are generally regarded as robust and numerically stable as they make use of computational techniques such as the QR factorization and the singular-value decomposition (SVR) [10].

\subsection{MPC using State-Space Models}

The availability of a dynamic model of the openloop process, for example in the form a discrete statespace model obtained through system identification approach provides the opportunity for the realisation of model predictive control (MPC). The objective with MPC at the current sampling time step $k$ is to bring the predicted output as close as possible to a specified target (denoted as $r(k)$ at time step $k$ ). This objective requires to identify the vector for the control input $\Delta U(k)$ over control horizon $N_{c}$,

$\Delta U(k)=\left[\Delta u(k)^{T}, \Delta u(k+1)^{T}, \ldots, \Delta u\left(k+N_{c}-1\right)^{T}\right]^{T}$,

so that the error function between the manipulated predicted output, $\hat{Y}(k)$, and the target output trajectory, $T(k)$, over the prediction horizon, $N_{p}$, is minimised:

$$
\begin{gathered}
J(k, \Delta U(k))=\|\widehat{Y}(k)-T(k)\|^{2}+ \\
+Q\|\Delta U(k)\|^{2} \rightarrow \min
\end{gathered}
$$

where

$$
\begin{aligned}
& \hat{Y}(k)=\left[\hat{y}(k+1)^{T}, \hat{y}(k+2)^{T}, \ldots, \hat{y}\left(k+N_{p}\right)^{T}\right]^{T} \\
& T(k)=\left[r(k+1)^{T}, r(k+2)^{T}, \ldots, r\left(k+N_{p}\right)^{T}\right]^{T}
\end{aligned}
$$

and the increment of a variable $v$ is

$$
\Delta v(k)=v(k)-v(k-1) .
$$

$Q>0$ is an $N_{c} \mathrm{x} N_{c}$ weighting matrix representing the relative importance of the manipulated (input) variables,

$$
Q=\left[\begin{array}{ccc}
q(1) & \cdots & 0 \\
\vdots & \ddots & \vdots \\
0 & \cdots & q\left(N_{c}\right)
\end{array}\right]
$$

The optimal vector of the manipulated variables that minimises the cost function $J$ is $\Delta U^{*}(k)$. For linear state-space model $\Delta U^{*}(k)$ can be found analytically. The control input value at step $k$ can then be calculated and used in the process control:

$$
u(k)=u(k-1)+\Delta u^{*}(k)
$$

\section{Study CaSe: 3D InkJet Printing of CONDUCTIVE LINES}

\subsection{Study Case Outline}

The study case for modelling the dynamic behaviour of 3D inkjet printing using state-space approach focus on the application of printing conductive lines (see 
Figure 4). The dynamic behaviour of interest is associated with monitoring and controlling a particular measurable process parameter, in this investigation this being the thickness of printed conductive lines. Dimensional and shape measurements performed inline are not imbedded in general in current 3D printers for electronics but there are already shifts towards making such capability available [7].

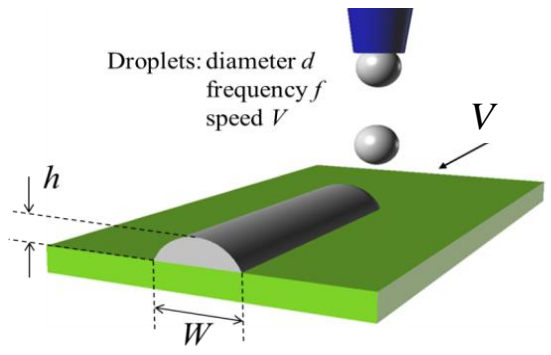

Fig. 4. Schematic of $3 \mathrm{D}$ inkjet printing of conductive lines.

Most piezo-electric drop-on-demand (DoD) printheads have integrated temperature control. Printhead temperature is one of the main process parameters that has direct influence on the ink viscosity. Varying temperature, either controlled or uncontrolled, affects the printing performance and the material characteristics, and therefore has an overall impact on the volume of ejected droplets and the quality of the printed features. Assuming in-line measurements of the thickness of printed lines over equally spaced sampling times are available through inspection system, it is feasible to imbed machine learning and realise process monitoring using model predictions for the dynamics of this, or other, product related parameter.

The ink material used in the study case is reported in reference [11]. The temperature dependent viscosity of this ink changes from $23.2 \mathrm{mPa} . \mathrm{s}$ at $60^{\circ} \mathrm{C}$ to 18.1 $\mathrm{mPa} . \mathrm{s}$ at $70^{\circ} \mathrm{C}$. The surface tension is found to vary little with temperature and hence assumed constant at $62.5 \mathrm{mN} / \mathrm{m}$. Experiments are performed to enable quantitative analysis of the droplet volume $\left(V_{\text {ool }} l_{\text {drop }}\right)$ and droplet velocity $\left(v_{\text {drop }}\right)$ at different printhead temperatures. The measurements are taken in the case of printhead with nozzle diameter $100 \mu \mathrm{m}$ and piezoelectric signal wave form with voltage amplitude $160 \mathrm{~V}$ and pulse duration $25 \mu \mathrm{sec}$. The following experimental data, gathered over the temperature range $60-70^{\circ} \mathrm{C}$ of the heated printhead, are adopted in this work:

- at $60^{\circ} \mathrm{C}: V_{\text {oldrop }}=179 \mathrm{pL}$ and $v_{d r o p}=1.9 \mathrm{~m} / \mathrm{sec}$;

- at $65^{\circ} \mathrm{C}: V_{\text {oro }}=200 \mathrm{pL}$ and $v_{\text {drop }}=2.5 \mathrm{~m} / \mathrm{sec}$;

- at $70^{\circ} \mathrm{C}: V_{\text {or }} l_{\text {drop }}=209 \mathrm{pL}$ and $v_{\text {drop }}=3.0 \mathrm{~m} / \mathrm{sec}$;
Due to unavailability of real measurements at present, the experimental data above is passed through a simulator of "measured" values for the thickness of printed conductive lines $(h)$. The data simulator uses the analytical model in Eq. 7 taken from reference [12]:

$$
h=\frac{\text { Vol drop }_{\text {drop }}}{d} \frac{f}{U}\left[\frac{W e+12}{3(1-\cos \theta)+\frac{4 W e}{\sqrt{R e}}}\right]^{-\frac{1}{2}}
$$

where

$$
\begin{array}{ll}
\text { Vol } l_{\text {drop }} & \text { : volume of a droplet } \\
f & : \text { printing frequency } \\
U & : \text { printhead scanning speed } \\
d & : \text { initial diameter of droplet } \\
\operatorname{Re} & : \text { Reynold number } \\
\text { We } & : \text { Weber number } \\
\theta & \text { : contact angle }
\end{array}
$$

Such model-generated values for given printhead temperature and associated ink viscosity/ droplet volume are considered as "measured" data. Measurement error is also introduced in the values of the thickness. In the following demonstration studies these values substitute the measurements that in real 3D inkjet printing equipment can be obtained from an inspection system.

\subsection{State-Space Model Development}

The state-space model is identified from data associated with a variable $\sin$-shape profile of the printhead temperature over the range $60^{\circ} \mathrm{C}$ to $68^{\circ} \mathrm{C}$. This profile is shown in Figure 5. The same figure details also the resulting profile of normalised values of conductive track (line) thicknesses, referred to as Process Run \#1, measured sequentially over equally spaced sampling intervals.

In the instance of Process Run \#1 data, no other factors or process variations affect the relationship between the printhead temperature and the associated thickness of the ink lines printed under specified (fixed) piezoelectric printhead attributes, wave form and respective ink properties. The varying printhead temperature affects the ink viscosity and hence under same piezoelectric pulse ejected droplets have different volume. Therefore, the resulting thickness of the printed tracks becomes variable.

The dataset associated with Process Run \#1 is used as training data to construct the state-space model, with model input $\boldsymbol{u}(\mathrm{t})$ the printhead temperature and model output $\boldsymbol{y}(\mathrm{t})$ the associated thickness of the printed conductive line (as with the model structure of Fig. 3). 


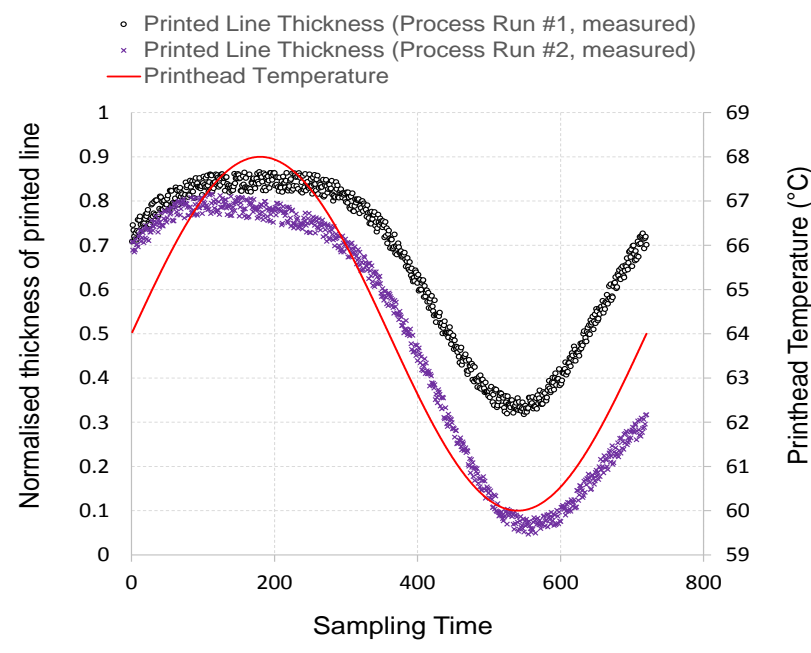

Fig. 5. Input (printhead temperature) and output (normalised thickness of conductive lines, Runs \#1 and \#2) process data.

The definition of the state-space model (model order 2) for the process of inkjet printing based on the process data detailed above is provided in Table 1 .

Table 1. Matrices and vectors defining the state-space model for the study case data.

\begin{tabular}{cc}
\hline \hline Model Entity & Vector/Matrix \\
\hline A & {$\left[\begin{array}{cc}0 & 1 \\
-0.9923 & 1.9922\end{array}\right]$} \\
\hline B & {$\left[\begin{array}{l}0.0003080 \\
0.0003066\end{array}\right]$} \\
\hline C & {$\left[\begin{array}{ll}1 & 0\end{array}\right]$} \\
\hline D & 0 \\
\hline K & {$\left[\begin{array}{l}1.006291 \\
1.073323\end{array}\right]$} \\
\hline
\end{tabular}

The initial state (X0) that minimises the prediction error of the developed state-space model is identified as the vector-column X0 $=\left[\begin{array}{ll}0.70993 & 0.69319\end{array}\right]^{\mathrm{T}}$.

\subsection{State-Space Model Validation}

The developed state-space process model is validated using the measured dataset from the Process Run \#2 (see Figure 5). A feature of this validation data is that the process input parameter, the printhead temperature, has the same sin-profile as the one used with Process Run \#1 but due to the effect of other implicit factors (e.g. ink printhead clogging, ambient and substrate temperature change, etc.) a progressive nonlinear reduction in the thickness of the printed lines takes effect.
Model validation and predictive performance are evaluated with three different prediction horizons: (1) 25 step-ahead, (2) 50 step-ahead and (3) 100 stepahead. In all cases, a FIT metric is used to indicate the accuracy of predictions over the range of validation data.

$$
F I T=100\left(1-\frac{\|\widetilde{\boldsymbol{y}}-\boldsymbol{y}\|}{\|\widetilde{\boldsymbol{y}}-\operatorname{mean}(\widehat{\boldsymbol{y}})\|}\right)
$$

where $\tilde{\boldsymbol{y}}$ is the measured output of the validation data and $\boldsymbol{y}$ is the respective model output.

Model validation results are shown in Figure 6. In all cases, particularly when the smaller 25 step-ahead horizon is monitored and forecasted, the different process dynamics of Process Run \#2 and the associated drift in process performance, indicated by the values of the thickness of printed lines, are accurately predicted.

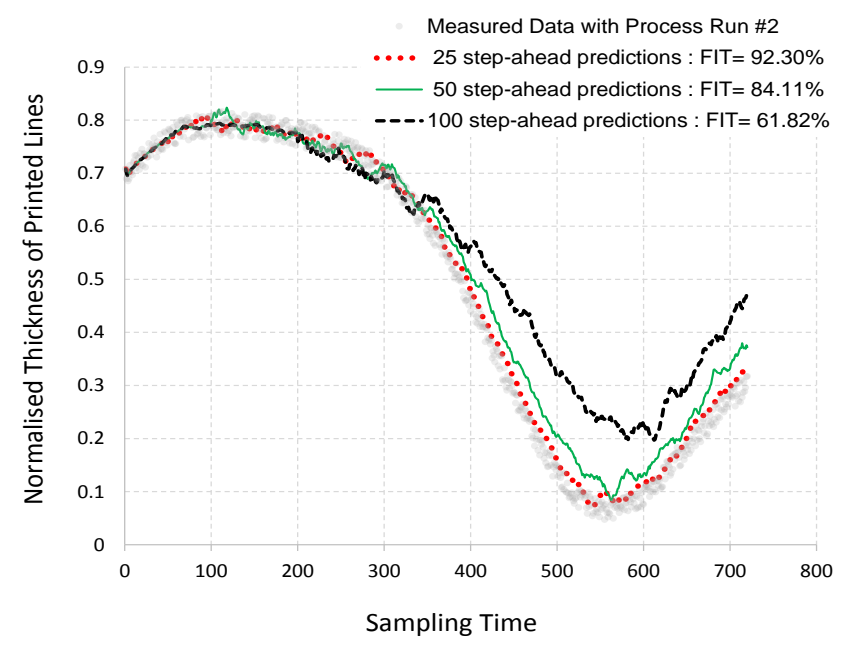

Fig. 6. Model validation with different prediction horizons and respective FIT measures for model predictive power and accuracy.

\subsection{MPC Demonstration}

Given the availability of a model of the dynamic behaviour of the process, the study case is also used to demonstrate the use of MPC methodology to achieve certain target for the measured thickness of printed lines. In this example, the initial state of the printhead is given with temperature $64^{\circ} \mathrm{C}$ and the aim of the process control is to achieve alternating normalised values of 0.7 and 0.6 of the thickness of the printed conductive lines which change from one level to the other every 100 units of the sampling time.

Figure 7 shows the required controlled change of the printhead temperature (bottom graph), assumed to be the control parameter, and based on the predicted 
process dynamics using the developed state-space model (Table 1). The graph at the top shows the statespace model predictions for the thickness of the printed conductive lines at each sampling point under the MPCidentified printhead temperature. In this demonstration, the MPC is implemented with predictive horizon $N_{p}=5$ and control horizon $N_{c}=5$. The weight matrix for the control parameter change has elements equal to 0.003 .
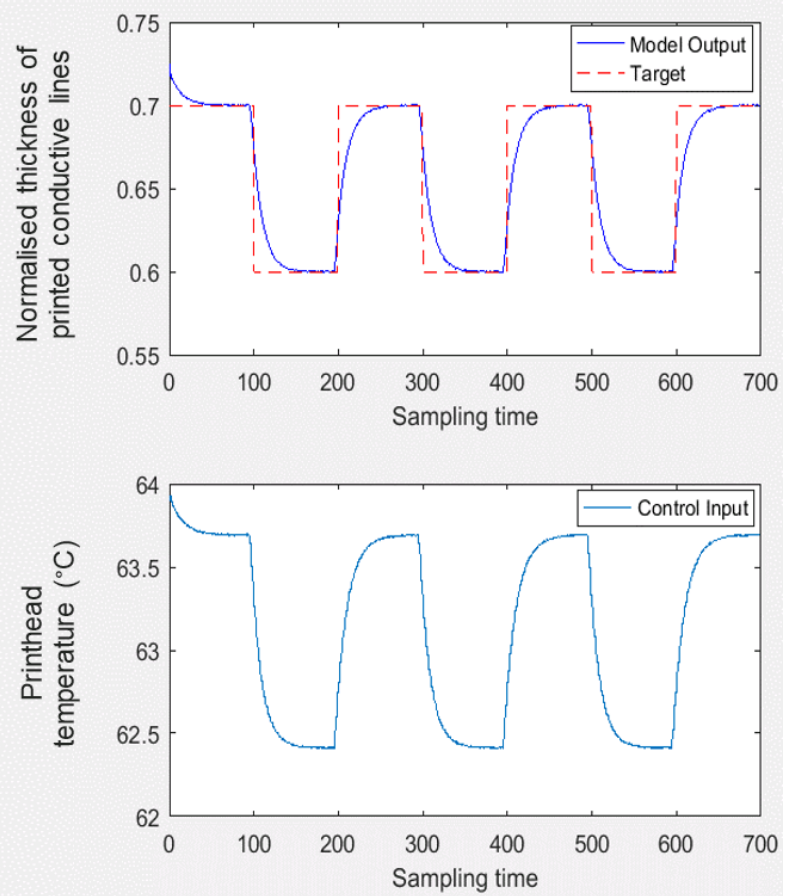

Fig. 7. Computed profile of the control parameter (printhead temperature) using MPC approach to achieve 2-level stepwise pattern for the thickness of printed lines.

\section{Conclusions}

Ongoing developments in 3D-Printing technologies and associated materials, and software tools for digital fabrication, have started to offer real prospects for their adoption by the electronics industry in high volume production lines. There are still challenges that need to be addressed to ensure that the quality and reliability of 3D-fabricated parts meet customer requirements. 3DPrinting process monitoring and in-line process control capabilities based on use of process data and machine learning models does not exist in present 3D-printers. This work has formulated and demonstrated the approach of development and use of state-space process models capable to capture accurately the dynamic behaviour of the printing process. The ability to perform predictive quality control on required dimensions and tolerances using model predictions has been discussed. Embedding such capabilities in real equipment can have big impact and has been advocated as having real potential to support wider technology acceptance for electronic manufacture and packaging as well as an improved, more robust process performance.

\section{ACKNOWLEDGMENTS}

The authors acknowledge the consortium in the EUfunded project NextFactory (http://www.nextfactoryproject.eu/), and European Community's 7th Framework (FP7/2007-2013) for funding this work under grant agreement No. 608985.

\section{REFERENCES}

[1] ASTM F2792 Standard, "Standard Terminology for Additive Manufacturing Technologies", available at: http://www.astm.org

[2] J. Salmeron et al. "Properties and printability of inkjet and screen-printed silver patterns for RFID antennas", Journal of Electronic Materials, Vol. 43, No. 2, 2014, pp. 604-617

[3] M. Mäntysalo "Evaluation of Inkjet Technology for Electronic Packaging and System Integration", Proc. 57th ECTC, NV, USA, May/June 2007, pp. 89-94

[4] H. Imai, S. Mizumo, A. Makabe, K. Sakurada, K. Wada "Applications of Inkjet Printing Technology to Electro Packaging", Proc 39th International Symposium on Microelectronics, San Diego, USA, 2006, pp. 484-490

[5] S.M. Bidoki et al. "Ink-jet Fabrication of Electronic Components", Journal of Micromechanics and Microengineering, Vol. 17, No. 5, 2007, pp. 967-974.

[6] Voxel 8, http://www.voxel8.com

[7] NextFactory, http://www.nextfactory-project.eu

[8] L. Ljung, "System Identification - Theory for the User", PTR Prentice Hall, Upper Saddle River, N.J., 1999, pp 132-134

[19 T. Soderstrom and P. Stoica, "System Identification", Publ. Prentice Hall, 1989

[10]M. Viberg "Subspace-based methods for the identification of linear time-invariant systems", Automatica, Vol. 31, No. 12, 1995, pp. 1835-1851

[11]D. Cibis and K. Kruger "Influencing Parameters in Droplet Formation for DoD Printing of Conductive Inks", Proc. 4th IMAPS/ACerS CICMT, Munich, Germany, April 2008, pp. 417-423.

[12] M. Pasandideh-Fard et al. "Capillary Effects during Droplet Impact on a Solid Surface", Phys. Fluids, Vol. 8, No. 3, 1996, pp. 650-659. 